\title{
On the Closed Hull of Subsets of Inductive Limits
}

\author{
By
}

\author{
Gerald HoFmanN*
}

\begin{abstract}
The present note investigates the closed hull of subsets $M$ contained in the (unrestricted) inductive limit $E[\tau]$ of topological vector spaces $E_{\alpha}\left[\tau_{\alpha}\right], \alpha \in \mathrm{A}$. Sufficient conditions for the validity of $\bar{M}^{\tau}=\cup_{\alpha \in \mathrm{A}} \overline{\mathrm{M} \cap E_{\alpha}} \tau_{\mathrm{n}}$ are given. These conditions are discussed by examples, and it is shown that there is not any redundance among them. Applications to F-linked topologies and sequence spaces are indicated. Futhermore, some inaccuracies contained in some of the standard text books are corrected.
\end{abstract}

\section{§1. Introduction}

For the following let us given a family $\left\{E_{\alpha}\left[\tau_{\alpha}\right]\right\}_{\alpha \in \mathrm{A}}$ of topological vector spaces such that i) there is a vector spaces $E$ with $E=\cup_{\alpha \in \mathrm{A}} E_{\alpha}$, ii) $\alpha \neq \beta$ implies $E_{\alpha} \neq E_{\beta}$, iii) the index set $\mathrm{A}$ is directed, where $\alpha<\beta$ if $E_{\alpha} \subset E_{\beta}$, iv) the embeddings $I_{\alpha, \beta}: E_{\alpha}\left[\tau_{\alpha}\right] \rightarrow E_{\beta}\left[\tau_{\beta}\right]$ are continuous for all $\alpha<\beta,(\alpha, \beta \in \mathrm{A})$. Let us mention that this is an often used realization for the construction of inductive limits.

The present note is aimed at an investigation of the closed hulls of subsets $M \subset E$, where $E$ is endowed with the vector topology of respectively, the unrestricted inductive limit $\tau_{u}$, the locally convex (l.c.) inductive limit $\tau_{i}$, and the weakest vector topology $\rho$ making the projections $P_{\alpha}: E[\rho] \rightarrow E_{\alpha}\left[\tau_{\alpha}\right], \alpha \in \mathrm{A}$, continuous (for a definition of $P_{\alpha}$ see (I), below). The main theorem gives sufficient conditions such that

$$
\bar{M}^{\rho}=\bar{M}^{\tau_{u}}=\bigcup_{\alpha \in \mathrm{A}} \overline{M \cap E_{\alpha}} \tau_{\mathrm{a}}
$$

and

$$
\bar{M}^{\tau_{u}}=\bar{M}^{\tau_{i}}
$$

respectively. These conditions are discussed by some examples, and it is shown that there is not any redundancy among them. Further, some applications of this theorem to the interesting concept of $F$-linked topologies are discussed. These results are finally applied to some problems of the theory of sequence spaces.

Communicated by H. Araki, November 20, 1990.

1991 Mathematics Subject Classification: 46A13.

* Univ. Leipzig, Sekt. Mathem., Augustusplatz-10, 7010 Leipzig, Germany. 
Let us mention that in some of the standard text books on topological vector spaces $([6],[8 \mathrm{a}])$ there are some inaccuracies concerning the closed hull of subsets of inductive limits. This is pointed out by some examples (see Remarks $3 b), 4 b$ ) below), and a correction is given in Theorem 1 and Corollary 1a).

The pattern of the present note is as follows. In $\$ 2$ the main theorem on the closed hull (Theorem 1) is stated and proved. The assumptions needed for the proof are discussed by Lemma 1 and Remark 2. The aim of $\S 3$ is to show that there is not any redundance among the assumptions of Theorem 1 . In $\S 4$, some applications to F-linked topologies on inductive limits are pointed out by Theorem 2 and Corollary 1. Finally, some applications to problems concerning sequence spaces are indicated in Corollary 2.

\section{§2. A Theorem on the Closed Hull}

Let us confirm some notation. For any (topological) vector space $X[\tau]$ and any linear subspace $Y \subset X$, let $\tau_{\uparrow Y}$ denote the topology which is induced by $\tau$ on the subspace $Y$. In the following let $\mathfrak{u}(\tau)$ stand for some (local) base of $O$ neighborhoods defining the (vector) topology $\tau$. Further, for any subset $Z \subset X$ let $\bar{Z}^{\tau}$ (resp. $\tilde{Z}^{\tau}$ ) be the closed hull of $Z$ in $X$ (resp. the completed hull of $Z$ ) with respect to $\tau$. Furthermore, if $\tau$ and $\tau^{\prime}$ are two given topologies on $X$, then let $\tau<\tau^{\prime}$ mean that $\tau^{\prime}$ is finer (stronger) than $\tau$.

Let $E=\bigcup_{\alpha \in \mathrm{A}} E_{\alpha}$ be endowed with respectively, the finest vector topology $\tau_{u}$ and the finest 1.c. topology $\tau_{i}$ such that the canonical embeddings

$$
I_{\alpha}: E_{\alpha}\left[\tau_{\alpha}\right] \rightarrow E\left[\tau_{u}\right]
$$

and

$$
I_{\alpha}: E_{\alpha}\left[\tau_{\alpha}\right] \rightarrow E\left[\tau_{i}\right]
$$

are continuous for all $\alpha \in \mathrm{A}$. Recall that $\tau_{u}$ and $\tau_{i}$ are the unrestricted inductive limit topology and the inductive limit topology, respectively (see $[14 ; 13.2 .4]$ ). Obviously, $\tau_{u}>\tau_{i}$. Furthermore, if the indexing set $\mathrm{A}$ is countable and $E_{\alpha}\left[\tau_{\alpha}\right]$, $\alpha \in \mathrm{A}$, are 1.c. spaces, then $\tau_{u}=\tau_{i}$, ([14; probl. 13.1.5, 13.1.108]).

Let us consider the following conditions: For each $\alpha \in \mathrm{A}$, let $E_{\alpha}$ be topologically complemented in $E\left[\tau_{u}\right]$ and $E\left[\tau_{i}\right]$, respectively, i.e., the canonical projections

$$
P_{\alpha}: E\left[\tau_{u}\right] \rightarrow E_{\alpha}\left[\tau_{\alpha}\right]
$$

and

$$
P_{\alpha}: E\left[\tau_{i}\right] \rightarrow E_{\alpha}\left[\tau_{\alpha}\right]
$$

( $\alpha \in \mathrm{A}$ ) are continuous. Recalling $\tau_{u}>\tau_{i},\left(\mathrm{I}^{\prime}\right)$ implies (I). Conditions (I), (I') are discussed by the following lemma. 
Lemma 1. a) (I) implies $\tau_{u \uparrow E_{\alpha}}=\tau_{\alpha}(\alpha \in \mathrm{A})$, and $\tau_{\alpha: E_{\beta}}=\tau_{\beta}$ for $\beta \in \mathrm{A}$, $\beta<\alpha$. If $E_{\alpha}\left[\tau_{\alpha}\right](\alpha \in \mathrm{A})$ are l.c. spaces and $\left(\mathrm{I}^{\prime}\right)$ is satisfied, then $\tau_{i \uparrow E_{\mathrm{a}}}=\tau_{\alpha}$ $(\alpha \in \mathrm{A})$, and $\tau_{\alpha ! E_{\beta}}=\tau_{\beta}$ for $\beta \in \mathrm{A}, \beta<\alpha$.

b) If $E_{\alpha}\left[\tau_{\alpha}\right], \alpha \in \mathrm{A}$, are l.c. spaces and (I) is satisfied, then ( $\left.I^{\prime}\right)$ follows.

c) Let us given a family of vector spaces (resp. l.c. spaces) $\left\{F_{\beta}\left[t_{\beta}\right] ; \beta \in \mathrm{B}\right\}$, $\mathrm{B}$ is a set of indices. Then, the unrestricted inductive limit $E\left[\tau_{u}\right]$ (resp. inductive limit $\left.E\left[\tau_{i}\right]\right)$ of $\left.\left\{E_{b}\left[\tau_{b}\right]\right) b \in \mathfrak{B}\right\}$ satisfies (I) (resp. (I')), where $\mathfrak{B}$ denotes the set of all the finite subsets of B directed by inclusion " $\subset$ ", and $E_{b}=\bigoplus_{\beta \in b} F_{\beta}\left[t_{\beta}\right]$.

d) Let $E\left[\tau_{u}\right]$ be the unrestricted inductive limit of a sequence of l.c. spaces $E_{n}\left[\tau_{n}\right]$, such that $E_{n+1} \supset E_{n}(n=0,1,2, \ldots), E=\bigcup_{n=0}^{\infty} E_{n}$, and (I) is satisifed. Then, $\tau_{u}=\tau_{i}$ and $E\left[\tau_{i}\right]=\bigoplus_{n=0}^{\infty} F_{n}\left[t_{n}\right]$, where $F_{0}\left[t_{0}\right]=E_{0}\left[\tau_{0}\right], F_{m}\left[t_{m}\right]=E_{m} / E_{m-1}\left[\widehat{\tau}_{m}\right]$, and $\widehat{\tau}_{m}$ denotes the quotient topology, $m=1,2,3, \ldots$

Proof. a) (I) and the continuity of $I_{\alpha}$ imply $\tau_{u\left\lceil E_{\mathrm{a}}\right.}>\tau_{\alpha}$ and $\tau_{\alpha}>\tau_{u}$ : $E_{\mathrm{a}}$, respectively. Thus, $\tau_{u \nmid E_{0}}=\tau_{\alpha}$ is implied. Now, let $\mathfrak{u}\left(\tau_{u}\right)$ be some base of 0 neighborhoods defining $\tau_{u}$ on $E$. Then, $\left\{U \cap E_{\alpha} ; U \in \mathfrak{u}\left(\tau_{u}\right)\right\}$ defines $\tau_{\alpha}$ on $E_{\alpha}$ $(\alpha \in \mathrm{A})$. For $\beta<\alpha(\alpha, \beta \in \mathrm{A})$, it follows that $\left(U \cap E_{\alpha}\right) \cap E_{\beta}=U \cap E_{\beta}$ and thus

$$
\tau_{\alpha \nmid E_{\beta}}=\tau_{u \uparrow E_{\beta}}=\tau_{\beta} .
$$

The assertions concerning $\tau_{i}$ follow analogously.

b) Recall that $\tau_{i}=\left(\tau_{u}\right)_{c}$, where $\left(\tau_{u}\right)_{c}$ denotes the finest 1.c. topology on $E$ weaker than $\tau_{u}$, and $\left(\tau_{u}\right)_{c}$ is defined by

$$
\mathfrak{U}\left(\left(\tau_{u}\right)_{c}\right)=\left\{\operatorname{aco}(U) ; U \in \mathfrak{H}\left(\tau_{u}\right)\right\},
$$

where aco (.) denotes the absolutely convex hull of., (see [14; 7.1.103, 13.1.101]). (I) implies now that for each absolutely convex $\tau_{\alpha}$-neighborhood of $0 V_{\alpha} \in \mathfrak{U}\left(\tau_{\alpha}\right)$, $\alpha \in \mathrm{A}$, there is a $U \in \mathfrak{H}\left(\tau_{u}\right)$ such that $P_{\alpha}(U) \subset V_{\alpha}$. Using that $V_{\alpha}$ is absolutely convex $P_{\alpha}(\operatorname{aco}(U)) \subset V_{\alpha}$ follows, Thus, $\left(I^{\prime}\right)$ is implied.

c) Consider the product

$$
G\left[\tau_{P}\right]=\prod_{\beta \in \mathrm{B}} F_{\beta}\left[t_{\beta}\right],
$$

and recall that for each $\beta \in \mathbf{B}, b \in \mathfrak{B}$, the canonical projections

$$
\hat{P}_{\beta}: G\left[\tau_{P}\right] \rightarrow F_{\beta}\left[t_{\beta}\right], \hat{P}_{b}: G\left[\tau_{P}\right] \rightarrow \prod_{\beta \in b} F_{\beta}\left[t_{\beta}\right]
$$

are continuous. Finally using $\tau_{P \nmid E}<\tau_{u}$ and $\prod_{\beta \in b} F_{\beta}\left[t_{\beta}\right]=\underset{\beta \in b}{\bigoplus} F_{\beta}\left[t_{\beta}\right]$, the continuity of $P_{a}=\hat{P}_{a \nmid E}: E\left[\tau_{u}\right] \rightarrow E_{\alpha}\left[\tau_{\alpha}\right]$ follows. Hence (I) is satisfied. The second assertion to be shown is now a consequence of $b$ ).

d) The observation made above implies $\tau_{u}=\tau_{i}$. Using now a) and the continuity of $P_{n}: E\left[\tau_{u}\right] \rightarrow E_{n}\left[\tau_{n}\right]$, the continuity of 


$$
P_{n+1, n}=P_{n \nmid \mathrm{E}_{n+1}}: E_{n+1}\left[\tau_{n+1}\right] \rightarrow E_{n}\left[\tau_{n}\right]
$$

is implied. Hence, the topological isomorphisms

$$
E_{n+1}\left[\tau_{n+1}\right] \cong E_{n}\left[\tau_{n}\right] \oplus F_{n}\left[t_{n+1}\right]
$$

follow, $n=0,1,2, \ldots$ Then,

$$
\begin{gathered}
E_{n}\left[\tau_{n}\right] \cong F_{0}\left[t_{0}\right] \oplus F_{1}\left[t_{1}\right] \oplus \ldots \oplus F_{n}\left[t_{n}\right], \\
E\left[\tau_{i}\right] \cong \bigoplus_{n=0}^{\infty} F_{n}\left[t_{n}\right]
\end{gathered}
$$

are implied, $([8 ; \S \S 18.5(2), 19.4])$. This completes the proof of Lemma 1.

Remark 1. Concerning the assertion of Lemma 1a) let us mention that if $\mathrm{A}=\mathbb{N}$ and $E_{n}\left[\tau_{n}\right]$ are 1.c. spaces $(n=0,1,2, \ldots)$, then $\tau_{i \uparrow E_{n}}=\tau_{n}$ are implied without assuming (I), (see [8]). However, if (I) is not satisfied and $A \neq \mathbb{N}$, then there are examples such that $\tau_{i \uparrow E_{\alpha_{0}}} \neq \tau_{\alpha_{0}}$ for some $\alpha_{0} \in \mathrm{A}$; (see [7]).

For the following let $\rho$ denote the weakest vector topology on $\mathbb{E}$ such that $P_{\alpha}: E[\rho] \rightarrow E_{\alpha}\left[\tau_{\alpha}\right], \alpha \in \mathrm{A}$, are continuous. Recall that $\rho$ is defined by the following base of O-neighborhoods

$$
\overline{\mathfrak{U}}(\rho)=\left\{P_{\alpha}^{-1}\left(U_{\alpha}\right) ; U_{\alpha} \in \mathfrak{U}\left(\tau_{\alpha}\right), \alpha \in \mathrm{A}\right\},
$$

where $\mathfrak{U}\left(\tau_{\alpha}\right)$ denotes some base of O-neighborhoods defining $\tau_{\alpha}$ on $E_{\alpha}$. Obviously (I) yields

$$
\rho<\tau_{u} \text {. }
$$

Notice also that if $E=\bigoplus_{\alpha \in \mathrm{A}} F_{\alpha}$, then $\rho$ is equivalent to the topology which is induced by the topology of the product $\prod_{\alpha \in \mathrm{A}} F_{\alpha}\left[\tau_{\alpha}\right]$ on its subspace $E$.

For a subset $M \subset E$ let us consider the following condition: There is an index $\alpha_{0} \in \mathrm{A}$ such that

$$
I_{\alpha} \circ P_{\alpha}(M) \subset M
$$

for all $\alpha \in \mathrm{A}$ with $\alpha>\alpha_{0}$.

Theorem 1 . Let us given the unrestricted inductive limit $E\left[\tau_{u}\right]$ of a family of topological vector spaces $\left\{E_{\alpha}\left[\tau_{\alpha}\right]\right\}_{\alpha \in \mathrm{A}}$ as in chapter 1 , and a subset $M \subset E$. Let (I) and (II) be satisfied.

a) It is

$$
\bar{M}^{\rho}=\bar{M}^{\tau_{u}}=\bigcup_{\alpha>\alpha_{0}} \overline{M \cap E_{\alpha}} \tau_{0} .
$$

b) If furthermore $E_{\alpha}\left[\tau_{\alpha}\right](\alpha \in \mathrm{A})$ are l.c. spaces and $\left(\mathrm{I}^{\prime}\right)$ is satisfied, then $\bar{M}^{\tau_{u}}=\bar{M}^{\tau_{i}}$ 
Proof. a) Using (1) and Lemma 1a), it follows that

$$
\cup_{\alpha>\alpha_{0}} \overline{M \cap E_{\alpha}} \tau_{\mathrm{n}} \subset \bar{M}^{\tau_{u}} \subset \bar{M}^{\rho} .
$$

Now, let us assume that there is an $f \in \bar{M}^{\rho}$ such that

$$
f \notin \underset{a>\alpha_{0}}{\cup} \overline{M \cap E_{\alpha}} \tau_{a} .
$$

Choose then an index $\beta \in \mathrm{A}$ with $\beta>\alpha_{0}$ and

$$
P_{\beta}(f)=f \text {. }
$$

Because of (3) there exists a O-neighborhood $U \in \mathfrak{l}\left(\tau_{\beta}\right)$ such that

$$
(f+U) \cap\left(M \cap E_{\beta}\right) \neq \varnothing .
$$

Further, using the continuity of $P_{\beta}: E[\rho] \rightarrow E_{\beta}\left[\tau_{\beta}\right]$, it follows that there is a $\mathrm{O}$ neighborhood $V \in \mathfrak{l}(\rho)$ with

$$
P_{\beta}(V) \subset U \text {. }
$$

Finally,

$$
P_{\beta}((f+V) \cap M) \subset P_{\beta}(f+V) \cap P_{\beta}(M) \stackrel{(1)}{\complement}(f+U) \cap\left(M \cap E_{\beta}\right) \stackrel{(4)}{=} \varnothing
$$

are implied, where $(*)$ is a consequence of $\left(3^{\prime}\right),\left(4^{\prime}\right)$, and (II). However $(f+$ $V) \cap M \neq \varnothing$, due to $f \in \bar{M}^{\rho}$ and $V \in \mathfrak{U}(\rho)$. This yields a contradiction to (5) since $P_{\beta}$ is defined on the whole of $\mathbb{E}$. Thus

$$
\bar{M}^{\rho} \subset \underset{a>a_{0}}{\cup} \overline{M \cap E_{a}} \tau_{a} .
$$

This implies together with (2) the assertion of a).

b) Noticing that the assumptions of b) imply $\rho<\tau_{i}$,

$$
\bar{M}^{\tau_{u}} \subset \bar{M}^{\tau_{t}} \subset \bar{M}^{\rho}
$$

follow. Applying a), the assertion under consideration is implied.

A discussion of (II) is given by the following.

Remark 2. If $\mathrm{A}=\mathbb{N}$, and $M$ is a linear subspace that satisfies (II), then there are linear subspaces $M_{0} \subset E_{0}, M_{n} \subset E_{n} / E_{n-1}(n=1,2,3, \ldots)$ such that

$$
M=M_{0} \oplus M_{1} \oplus M_{2} \oplus \ldots
$$

Proof. Let us put

$$
\begin{aligned}
& M_{0}=\left\{P_{0}(f) ; f \in M\right\}, \\
& M_{n}=\left\{P_{n}(f)-P_{n-1}(f) ; f \in M\right\},
\end{aligned}
$$

$n=1,2,3, \ldots$ The assumptions of the assertion under consideration imply 
$M_{m} \subset M(m=0,1,2, \ldots)$. Using the isomorphisms considered in the proof of Lemma 1d), let $M_{n}$ be canonically identified with a linar subspace of $F_{n}=E_{n}$ l $E_{n-1}$. Hence,

$$
\bigoplus_{m=0}^{\infty} M_{m} \subset M .
$$

Conversely, let $f \in M$. Considering $f_{0}=P_{0}(f) \in M_{0}, f_{n}=P_{n}(f)-P_{n-1}(f) \in M_{n}$ $(n=1,2,3, \ldots)$, it follows that

$$
f=f_{0} \oplus f_{1} \oplus f_{2} \oplus \ldots \in \bigoplus_{m=0}^{\infty} M_{m} .
$$

\section{$\S 3 . \quad$ Discussion of Theorem 1}

It will be shown by two examples that there is not any redundance among the assumptions (I), (II) of Theorem 1.

Example 1. Let us given a strict inductive limit

$$
E[\tau]=\lim _{\rightarrow} E_{n}\left[\tau_{n l}\right]
$$

with a defining sequence $\left(E_{n}\left[\tau_{n}\right]\right)_{n=1}^{\infty}$ of 1.c. spaces satisfying $E_{n} \subset E_{n+1}, E=$ $\bigcup_{n=1}^{\infty} E_{n}$, and $\tau_{n+1} \uparrow E_{n}=\tau_{n}, n=1,2,3, \ldots$. Further, let us assume that $E[\tau]$ is not a direct sum as described in Lemma 1d). (For a concrete example one can choose the Schwartz-spaces $\mathscr{D}$ of basic functions having a compact support.) Notice that (I) is not satisfied. Then there is a subsequence of $\left(E_{n}\right)_{n=1}^{\infty}$ such that $P_{m}$ : $E[\tau] \rightarrow E_{m}\left[\tau_{m}\right]$ are not continuous for all $m \in \mathbb{N}$. Hence there is a net $\left(v^{(\beta)}\right)_{\beta \in \mathbb{B}}$, $\mathrm{B}$ is a directed set of indices, $v^{(\beta)} \in E$, such that

$$
v^{(\beta)} \rightarrow 0
$$

concerning $\tau$, and for each $m \in \mathbb{N}$,

$$
P_{m}\left(v^{(\beta)}\right) \nrightarrow 0
$$

concerning $\tau_{m}$. Consider the set

$$
M=\left\{v^{(\beta)} ; \beta \in \mathrm{B}\right\} \cup\left\{P_{1}\left(v^{(\beta)}\right) ; \beta \in \mathbb{B}\right\} \cup\left\{P_{2}\left(v^{(\beta)}\right) ; \beta \in \mathrm{B}\right\} \cup \ldots
$$

Then,

i) $P_{n}(M) \subset M, n=1,2,3, \ldots$,

ii) $0 \in \bar{M}^{\tau}, \quad$ iii) $0 \notin \bigcup_{n=1}^{\infty} \overline{M \cap E_{n}} \tau_{n}$

follow. Thus the assertion of Theorem 1a) does not hold true. This example shows that (I) is not any redundance among the assumptions of Theorem 1a). 
Example 2. Consider the direct sum

$$
\mathbf{C}_{\otimes}=\mathbf{C} \oplus \mathbf{C} \oplus \ldots,
$$

where $\mathbf{C}$ stands for the set of complex numbers. Further, let

$$
\mathbf{C}_{\mathbf{N}}=\mathbf{C} \oplus \mathbf{C} \oplus \ldots \oplus \mathbf{C}(\mathrm{N}+1 \text { items }), N \in \mathbb{N}^{*},
$$

denote the $N^{\text {th }}$ "truncated" part of $\mathbf{C}_{\otimes}$. In the following let $\mathbf{C}_{\otimes}$ be furnished with the topology of the direct sum $\tau$, i.e., $\tau$ is defined by the following system of semi-norms

$$
\left\{f \rightarrow p_{\left(r_{n}\right)}(f) ;\left(r_{n}\right)_{n=0}^{\infty} \in \mathbf{R}_{+}^{\mathbf{N}^{+}}\right\}
$$

where

$$
p_{\left(r_{n}\right)}(f)=\sum_{n=0}^{\infty} r_{n}\left|f_{n}\right|,
$$

$f=\left(f_{0}, f_{1}, \ldots, f_{m}, 0,0, \ldots\right) \in \mathbf{C}_{\otimes}$, and $\mathbf{R}_{+}^{\mathbf{N}^{+}}$denotes the set of all the sequences of positive real numbers. (Recalling that for all but finitely many homogeneous components $f_{n}$ of $f \in \mathbf{C}_{\otimes}$ the equality $f_{n}=0$ holds, (6) is well-defined.)

Furthermore, let $\tau_{N}$ stand for the 1.c. topology on $\mathbf{C}_{N}$ that is induced by $\tau$ on the subspace $\mathbf{C}_{N}$ of $\mathbf{C}_{\otimes}$. Notice that $\mathbf{C}_{\otimes}[\tau]$ is a strict inductive limit with the defining sequence $\left(\mathbf{C}_{N}\left[\tau_{N}\right]\right)_{N=0}^{\infty}$, i.e.,

$$
\mathbf{C}_{\otimes}=\lim _{\rightarrow} \mathbf{C}_{N}\left[\tau_{N}\right]
$$

Now, let us consider the set

$M=\left\{f \in \mathbf{C}_{\otimes} ; f=f_{0} \oplus f_{n}, f_{0}, f_{\mathrm{n}} \neq 0, n\left(\left|f_{0}\right|+\left|f_{n}\right|\right) \geq 1, n=1,2, \ldots\right\}$, where $f_{0} \oplus f_{n}=$ $\left(f_{0}, 0, \ldots, 0, f_{n}, 0,0, \ldots\right)$.

Lemma 2. The following are satisfied:

a) $M$ does not satisfy condition (II),

b) $0 \notin \bigcup_{N=0}^{\infty} \overline{M \cap \mathbf{C}_{N}} \tau_{N}$,

c) $0 \in \bar{M}^{\tau}$

Proof. a) Take any $f_{0} \oplus f_{n} \in M$ and consider $P_{n-1}\left(f_{0} \oplus f_{n}\right)=f_{0}$. Noticing that every $f \in M$ has exactly two non-vanishing homogeneous components, a) follows.

b) Assume that b) is not satisfied. Then there is a certain $n^{\prime} \in \mathbb{N}$ such that

$$
0 \in \overline{M \cap \mathbb{C}_{n}} \tau_{n}
$$

Hence, for every sequence $\left(r_{n}\right)_{n=0}^{\infty} \in \mathbb{R}_{+}^{\mathbf{N}^{+}}$and every $\varepsilon>0$ there has to be an

$$
f=f_{0} \oplus f_{m} \in M \cap \mathbb{C}_{n^{\prime}}
$$


with

$$
p_{\left(r_{n}\right)}(f) \leq \varepsilon
$$

Note that (7) implies $m \leq n^{\prime}$. Choosing $\varepsilon=\frac{1}{2 n^{\prime}}, r_{0}=r_{1}=\ldots=1$, (8) implies

$$
\frac{1}{2} \geq n^{\prime}\left(\left|f_{0}\right|+\left|f_{m}\right|\right) \geq m\left(\left|f_{0}\right|+\left|f_{m}\right|\right)
$$

Thus $f \notin M$ follows. But this contradicts (7).

c) Let us given any $\left(r_{n}\right)_{n=0}^{\infty} \in \mathbf{R}_{+}^{\mathbf{N}^{*}}, \varepsilon>0$. Then choose $n^{\prime} \in \mathbf{N}$ with $n^{\prime}>$ $2 r_{0} / \varepsilon$. Consider $f=f_{0} \oplus f_{n^{\prime}}$, where $f_{0}=\varepsilon / 2 r_{0}, f_{n^{\prime}}=\varepsilon / 2 r_{n^{\prime}}$. Because of

$$
n^{\prime}\left(\left|f_{0}\right|+\left|f_{n^{\prime}}\right|\right)=\frac{n^{\prime} \varepsilon}{2}\left(1 / r_{0}+1 / r_{n^{\prime}}\right)>r_{0}\left(1 / r_{0}+1 / r_{n^{\prime}}\right)>1,
$$

$f \in M$ follows. Then,

$$
p_{\left(r_{n}\right)}(f)=r_{0}\left|f_{0}\right|+r_{n^{\prime}}\left|f_{n^{\prime}}\right|=\varepsilon / 2+\varepsilon / 2=\varepsilon
$$

yields c).

Remark 3. a) Because of Lemma 1c), the present example satisfies assumption (I). Hence, Lemma 2 implies that (II) is not any redundance among the assumptions of Theorem 1.

b) Example 2 sets also a counter-example to $[8 \mathrm{a} ; \S 19.5(5),(6)]$. Hence these assertions are incorrect, and Theorem 1 represents a possibility to correct them. Let us mention that the last edition [8] does not contain any assertions about the closed hull of subsets of inductive limits.

c) In the case of tensor-algebras, condition (II) was first considered by J. Yngvason ([4], [15]).

\section{§4. On F-linked Topologies on Inductive Limits}

Following Wilansky, let us recall the concept of F-linked topologies.

Definition $\mathbb{1}([14 ; 6.1 .9])$. Let $t, t^{\prime}$ be vector topologies for a vector space $X$. Then, $t^{\prime}$ is called F-linked to $t$ if there is a base of O-neighborhoods $\mathfrak{u}\left(t^{\prime}\right)$ which defines $t^{\prime}$ and each $U \in \mathfrak{l}\left(t^{\prime}\right)$ is $t$-closed.

Remember also that among others there are the following interesting applications of this concept: i) characterization of completed and closed hulls ([8; $\$ 18.4(4)],[3],[14 ; 6.1 .13,6.1 .16])$, ii) characterization of polar topologies ([14; probl. 8.5.4]), and iii) characterizations of barrelled and ultrabarrelled vector spaces $([14$; probl. 9.3.112-114, 126, 131]). 
Definition 2. Let us given $E=\bigcup_{\alpha \in \mathrm{A}} E_{\alpha}$ as in chapter 1, and let (I) be satisfied. Then, a test topology $t$ on $E$ (i.e., $I_{\alpha}: E_{\alpha}\left[\tau_{\alpha}\right] \rightarrow E[t], \alpha \in \mathrm{A}$, are continuous, [14; 13.1.1]), is called filtrated, if

i) there is a $t$-defining base $\mathfrak{u}(t)$ of $\mathrm{O}$-neighborhoods with the property: For each $U \in \mathfrak{U}(t)$ there is an index $\alpha_{0} \in \mathrm{A}$ such that $I_{\alpha} \circ P_{\alpha}(U) \subset U$ for all $\alpha>\alpha_{0}$,

ii) $\rho<t$.

Lemma 3. Let $E\left[\tau_{u}\right]$ satisfy (I). Then,

a) $\rho$ is the weakest filtrated topology on $E$,

b) every vector topology $t$ with $\rho<t<\tau_{u}$ satisfies

$$
t_{\uparrow E_{\mathrm{a}}}=\tau_{\alpha}, \quad \alpha \in \mathrm{A} .
$$

Proof. a) Using (1), it is implied that $\rho$ is a test topology. Further, consider the base of O-neighborhoods

$$
\mathfrak{u}(\rho)=\left\{P_{\alpha}^{-1}\left(U_{\alpha}\right) ; U_{\alpha} \in \mathfrak{u}\left(\tau_{\alpha}\right), \alpha \in \mathrm{A}\right\}
$$

which defines $\rho$ on $E$. For $U=P_{\beta}^{-1}\left(U_{\beta}\right) \in \mathfrak{u}(\rho)$, choose $\alpha_{0}=\beta$. For each $\alpha \in \mathrm{A}$, $\alpha>\alpha_{0}$, it follows

$$
\begin{gathered}
U=U_{\beta} \oplus\left(E_{\alpha} / E_{\alpha_{0}}\right) \oplus\left(E / E_{\alpha}\right), \\
I_{\alpha}^{\prime} \circ P_{\alpha}(U)=I_{\alpha}\left(U_{\beta} \oplus\left(E_{a} / E_{\alpha_{01}}\right) \subset U .\right.
\end{gathered}
$$

This proves a).

b) Using the continuity of $P_{\alpha}: E[\rho] \rightarrow E_{\alpha}\left[\tau_{\alpha}\right]$, (1) and Lemma 1a), the assertion under consideration is implied by

$$
\tau_{\alpha}<\rho_{\uparrow E_{\mathrm{a}}}<t_{\uparrow E_{\mathrm{a}}}<\tau_{u \uparrow E_{\mathrm{a}}}=\tau_{\alpha}
$$

$\alpha \in \mathrm{A}$.

Theorem 2. Let us given the unrestricted inductive limit $E\left[\tau_{u}\right]$. If $E\left[\tau_{u}\right]$ satisfies (I) and $t^{\prime}$ is a filtrated topology on $E$, then $t^{\prime}$ is F-linked to every vector topology $t$ that satisfies $\rho<t<\tau_{u}$.

Proof. Definition 2 and Lemma 3a) imply $\rho<t^{\prime}<\tau_{u}$. Consider the base $\mathfrak{u}\left(t^{\prime}\right)$ given in Definition 2 , and notice that

$$
\begin{aligned}
& \bar{U}^{\rho} \supset \bar{U}^{t^{\prime}} \supset \bar{U}^{\tau_{u}}, \\
& \bar{U}^{\rho} \supset \bar{U}^{t} \supset \bar{U}^{\tau_{u}},
\end{aligned}
$$

for each $U \in \mathfrak{l}\left(t^{\prime}\right)$. Applying Theorem 1a) to $M=U$, it follows that $\bar{U}^{t}=\bar{U}^{t^{\prime}}$. Finally noticing that $\left\{\bar{U}^{t} ; U \in \mathfrak{U}\left(t^{\prime}\right)\right\}$ defines $t^{\prime}$, the proof is completed.

Let us discuss Theorem 2 for the special case of the direct sums $E\left[\tau_{u}\right]$ and $E\left[\tau_{i}\right]$ of vector spaces and 1.c. spaces $F_{\beta}\left[t_{\beta}\right], \beta \in \mathrm{B}$ (set of indices), respectively. 
Lemma 4. Let us given vector spaces (resp. l.c. spaces) $F_{\beta}\left[t_{\beta}\right], \beta \in \mathrm{B}$. Then,

a) $\tau_{u}\left(\right.$ resp. $\left.\tau_{i}\right)$ is the finest filtrated vector topology (resp. finest filirated l.c. topology) on $E=\bigoplus_{\beta \in \mathrm{B}} F_{\beta}$,

b) the box topology $\gamma$ is also filtrated.

Proof. a) Using Lemma 1c) and (1), it follows that Definition 2ii) applies to $\tau_{u}$ and $\tau_{i}$, respectively. Let $\mathfrak{u}\left(t_{\beta}\right)$ be bases of O-neighborhoods which define $t_{\beta}$ on $F_{\beta}, \beta \in \mathrm{B}$ Then,

$$
\mathfrak{U}\left(\tau_{b}\right)=\left\{V_{b} \subset E_{b} ; \text { there are } \beta \in b, U_{\beta} \in \mathfrak{u}\left(t_{\beta}\right) \text { such that } V_{b}=P_{b, \beta}^{-1}\left(U_{\beta}\right)\right\}
$$

defines $\tau_{b}$ on $E_{b}$, where $P_{b, \beta}=P_{\beta \uparrow E_{b}}, P_{\beta}: E \rightarrow F_{\beta}$ (canonical projection). Remembering that the system of all

$$
U=\bigcup_{k=1}^{\infty} \sum_{n=1}^{k} \bigcup_{b \in \mathfrak{B}} I_{b}\left(V_{b}^{(n)}\right)
$$

$V_{b}^{(n)} \in \mathfrak{l}\left(\tau_{b}\right), n=1,2,3, \ldots$, defines $\tau_{u}$ on $E$, condition i) of Definition 2 follows from

$$
\begin{gathered}
I_{b_{0}} \circ P_{b_{0}}(U) \subset \bigcup_{k=1}^{\infty} \sum_{n=1}^{k} \cup_{b \in \mathfrak{B}} I_{b_{0}} \circ P_{b_{0}} \circ I_{b}\left(V_{b}^{(n)}\right) \\
=\bigcup_{k=1}^{\infty} \sum_{n=1}^{n} \bigcup_{b \in \mathfrak{B}} I_{c} \circ P_{b, c}\left(V_{b}^{(n)}\right) \subset U
\end{gathered}
$$

for all $b_{0} \in \mathfrak{B}$, where $P_{b, c}=P_{c} \uparrow E_{b}, P_{c}: E \rightarrow E_{c}$ (canonical projection), $c=b \cap$ $b_{0} \in \Re, P_{b, \varnothing}\left(V_{b}^{(n)}\right)=0$. Finally recalling that $\tau_{u}$ is the finest test topology on $E$, the assertion under consideration is shown for $\tau_{u}$.

Now, let $F_{\beta}\left[t_{\beta}\right], \beta \in \mathbb{B}$, be 1.c. spaces. Recall that $\tau_{i}$ is defined by

$$
\mathfrak{u}\left(\tau_{i}\right)=\left\{\operatorname{aco}(U) ; U \in \mathfrak{U}\left(\tau_{u}\right)\right\} .
$$

If $u \in \operatorname{aco}(U)=\left\{\lambda u_{1}+\mu u_{2} ;|\lambda|+|\mu| \leq 1, u_{1}, u_{2} \in U\right\}$, then

$$
I_{\alpha} \circ P_{\alpha}(u)=\lambda I_{\alpha} \circ P_{\alpha}\left(u_{1}\right)+\mu I_{\alpha} \circ P_{\alpha}\left(u_{2}\right) \in \operatorname{aco}(U)
$$

because of $I_{\alpha} \circ P_{\alpha}\left(u_{j}\right) \in U(j=1,2), \alpha \in \mathrm{A}$.

b) Recalling that $\gamma$ is defined by

$$
\mathfrak{U}(\gamma)=\left\{\bigoplus_{\beta \in B} U_{\beta} ; t_{\beta} \in \mathfrak{H}\left(t_{\beta}\right)\right\},
$$

and it satisfies $\rho<\gamma<\gamma_{u}$, the filtratedness of $\gamma$ follows straightforwardly.

Corollary 1. Assume that the assumption of Lemma 4 are satisfied. Further let us given a filtrated vector topology $\tau^{\prime}$ on $E$.

a) $\tau^{\prime}$ is F-linked to every vector topology $\tau$ on $E$ which satisfies $\rho<\tau<\tau_{u}$. 
b) If $E\left[\tau^{\prime}\right]$ is ultrabarrelled, then $\tau^{\prime}=\tau_{u}$.

c) If $E_{\beta}\left[t_{\beta}\right], \beta \in \mathrm{B}$, are l.c. spaces and $E\left[\tau^{\prime}\right]$ is barrelled, then $\tau^{\prime}=\tau_{i}$.

d) If $\tau^{\prime \prime}$ is a second filtrated topology on $E$ such that $\tau^{\prime \prime}<\tau^{\prime}$, then $\tilde{E}^{\rho} \supset \tilde{E}^{\tau^{\prime \prime}}$ $\supset \tilde{E}^{\tau^{\prime}} \supset \tilde{E}^{\tau_{u}}$.

Proof. a) follows from Lemma 1c) and Theorem 2.

b) follows from Lemma 4 and [14; probl. 9.3.113].

c) follows from Lemma 4 and [14; probl. 9.3.112].

d) is a consequence of the closed neighborhood theorem, Theorem 2 and Lemma 4.

Remark 4. a) If $\tau^{\prime}=\tau_{u}, \tau=\rho$, then Corollary 1a) implies the assertion of $[14 ; 13.2 .11]$.

b) The following Example 3 shows that the assertion stated in $[6 ; 4.4 .2]$ is incorrect. However, setting $\tau=\rho$, Corollary 1a) gives a correction of this assertion.

c) For tensor-algebras, some families of filtrated 1.c. topologies are introduced and investigated, e.g., in [2], [4], [5], [9], [12], [15]. Let us mention that the investigations of tensor-algebras were initiated by Borchers and Uhlmann ([1], [13]).

The aim of the following is to show that the assumption of the filtratedness of $\tau^{\prime}$ is not any redundancy in Theorem 2 and Corollary 1 .

Example 3. Let us consider the Schwartz space $\mathscr{S}(\mathbf{R})$ of basic functions endowed with its well-known 1.c. topology given by the system of norms

$$
p^{(m)}(h)=\left(\int_{-\infty}^{\infty}\left|N^{m} h(x)\right|^{2} d x\right)^{1 / 2},
$$

$m=0,1,2, \ldots$, where $N=1+x^{2}-\left(d^{2} / d x^{2}\right), h \in \mathscr{Y}(\mathbf{R})$, (e.g., see [10]). Let

$$
E=\bigoplus_{n=1}^{\infty} F_{n}
$$

where $F_{n}=\mathscr{Y}(\mathbb{R})$. On $E$ let us consider the l.c. topologies $\tau_{u}, \tau_{\propto}$, and $\rho$ given by the following systems of semi-norms:

$\tau_{u}: \quad p_{\left(\gamma_{n}\right)\left(m_{n}\right)}(f)=\sum_{n=1}^{\infty} \gamma_{n} p^{\left(m_{n}\right)}\left(f_{n}\right),\left(\gamma_{n}\right),\left(m_{n}\right) \in \mathbf{N}^{\mathbf{N}}$ (set of all the sequences of natural numbers),

$\tau_{\infty}: \quad p_{\left(\gamma_{n}\right), m}(f),\left(\gamma_{n}\right) \in \mathbf{N}^{\mathbf{N}}, m=0,1,2, \ldots$ (fixed in each semi-norm!),

$\rho: \quad q_{n, k}(f)=p^{(n)}\left(f_{k}\right), n, k=0,1,2, \ldots$,

where $f=\left(f_{1}, f_{2}, \ldots, f_{N}, 0,0, \ldots\right) \in E$. Note that $E$ is complete with respect to both $\tau_{\infty}$ and $\tau_{u}$. 
Consider now the linear functional $T(f)=\sum_{n=1}^{\infty} T_{n}\left(f_{n}\right)$ where

$$
T_{n}\left(f_{n}\right)=\int_{-\infty}^{\infty}(d / d x)^{n} f_{n}(x) d x .
$$

Notice that $T$ is $\tau_{u}$-continuous, but not $\tau_{\infty}$-continuous. Furthermore, let us consider the l.c. topology $\tau^{*}$ defined by the semi-norms

$$
\left\{p_{\left(\gamma_{n}\right), m} ;\left(\gamma_{n}\right) \in \mathbf{N}^{\mathbf{N}}, m \in \mathbf{N}\right\} \cup\left\{p_{T}\right\},
$$

where $p_{T}(f)=|T(f)|, f \in E$. Obviously,

$$
\tau_{\infty}<\tau^{*}<\tau_{u}
$$

Lemma 5. $E$ is not complete concerning $\tau^{*}$.

Proof. Let us assume the contrary. Since $\mathrm{T}$ is not $\tau_{\infty}$-continuous, the hyperplane

$$
H=\{f \in E: T(f)=1\}
$$

is dense in $E$ concerning $\tau_{\infty}$. Hence there is a net $\left(f^{(\mu)}\right)_{\mu \in \Delta} f^{(\mu)} \in H, \Delta$ is a directed set of indices, such that

$$
f^{(\mu)} \rightarrow 0
$$

with respect to $\tau_{x}$. Now, (9) and $p_{T}\left(f^{(\mu)}-f^{\left(\mu^{\prime}\right)}\right)=0, \mu, \mu^{\prime} \in \Delta$, imply that $\left(f^{(\mu)}\right)_{\mu \in \Delta}$ is a Cauchy-net with respect to $\tau^{*}$. Using our assumption, the existence of $g \in E$ satisfying $f^{(\mu)} \rightarrow g$ with respect to $\tau^{*}$ is implied. Because of $\tau_{\infty}<\tau^{*}$, it follows that $f^{(\mu)} \rightarrow g$ holds also with respect to $\tau_{\infty}$. However, this yields $g=0$ being a contradiction to

$$
P_{T}\left(f^{(\mu)}\right)=\left|T\left(f^{(\mu)}\right)\right|=1
$$

$\mu \in \Delta$. Thus the proof is completed.

Notice that condition i) of Definition 2 does not apply to

$$
U=\left\{f \in E ; p_{T}(f) \leq 1\right\} .
$$

Recalling that $E$ is complete with respect to both $\tau_{\infty}$ and $\tau_{u}$, the closed neighborhood theorem and Lemma 5 imply that $U$ is not closed concerning both $\rho$ and $\tau_{\infty}$. Hence, the assumption of the filtratedness is not redundant in Corollary 1 . Furthermore, it follows that $\tau^{*}$ is not F-linked to $\rho$. Thus the assertion of $[6 ; 4.4 .2]$ is incorrect. 
Let us give some simple applications of the foregoing to the following typical problems concerning sequence spaces: a) problem of identification, b) inclusion problem (Abelian theorems), c) intersection problem (Tauberian theorems, i.e., are there sequence spaces $S_{j}, j=1,2,3$, such that $S_{1} \supset S_{2}$ and $S_{1} \cap S_{3} \subset S_{2}$ ?), see $[11 ;$ p.2].

Let us put $\mathrm{B}=\mathbf{N}$ and $F_{\beta}=\mathbf{C}$ (endowed with its standard topology) in Lemma 4 . Then, $E=\varphi$ (space of complex sequences which are eventually zero) and $\tau_{u}=\tau_{i}$.

Corollary 2. a) If $\tau$ is a filtrated topology for $\varphi$, then $\tilde{\varphi}^{\tau}$ is a sequence space.

b) If $\tau_{j}(j=1,2)$ are filtrated topologies for $\varphi$ such that $\tau_{1}<\tau_{2}$, then the sequence spaces $\tilde{\varphi}^{\tau_{l}}$ satisfy $\tilde{\varphi}^{\tau_{2}} \subset \tilde{\varphi}^{\tau_{1}}$.

c) Let us given filtrated topologies $\tau_{j}(j=1,2,3)$ such that $\tau_{1}<\tau_{2}<\sup \left(\tau_{1}\right.$, $\left.\tau_{3}\right)$. Then,

$$
\tilde{\varphi}^{\tau_{1}} \cap \tilde{\varphi}^{\tau_{i}} \subset \tilde{\varphi}^{\tau_{2}}
$$

Proof. a), b) Noticing that $\omega=\tilde{\varphi}^{\rho}$ (space of all complex sequences), Corollary 1d) implies the assertions of a), b).

c) Recalling that $\mathfrak{u}\left(\sup \left(\tau_{1}, \tau_{3}\right)\right)=\left\{U_{1} \cap U_{3} ; U_{j} \in \mathfrak{H}\left(\tau_{j}\right), j \in\{1,3\}\right\}$, the filtratedness of $\sup \left(\tau_{1}, \tau_{3}\right)$ is implied. The assertion under consideration is now a consequence of $\tilde{\varphi}^{\tau_{1}} \cap \tilde{\varphi}^{\tau_{z}}=\tilde{\varphi}^{\sup \left(\tau_{1}, \tau_{\xi}\right)}\left(\left[14 ;\right.\right.$ probl. 6.1.4]), $\tau_{2}<\sup \left(\tau_{1}, \tau_{3}\right)$, and Corollary 1d).

Let us mention that the method of constructing new sequence spaces by completion of $\varphi$ is also used by Dubin and Hennings ([2]).

\section{References}

[1] Borchers, H.J., On the structure of the algebra of field operators, Nuovo Cimento, 24 (1962), 214-236.

[2] Dubin, A.D. and Hennings, M.A., Regular tensor algebras, Publ. RIMS Kyoto Univ., 25 (1989), 971-999.

[3] Garling, D.J.H., The filter condition, the closed neighbourhood condition and consistent seminorms, Duke Math. J., 38 (1971), 299-304.

[4] Hofmann, G., Die Testfunktionenalgebra $\mathscr{S}_{\otimes}$ und ihre Anwendung in der axiomatischen Quantenfeldtheorie, Diss. A, Leipzig, 1975.

[5] Topological tensor-algebras. I. Properties of intermediate locally convex topologies, preprint, Leipzig (1989).

[6] Jarchow, H., Locally convex spaces, Teubner, Stuttgart, 1981.

[7] Komura, Y., Some examples in topological spaces, Math. Ann., 153 (1964), 150-162.

[8] Köthe, G., Topological vector spaces I, Springer-Verl., Berlin, New York, Heidelberg, 1983.

[8a] _ Topologische lineare Räume I, Springer-Verl., Berlin, Göttingen, Heidelberg, 1960.

[9] Laßner, G., On the structure of the rest function algebra, Dubna preprint, JINR, E2-5254 (1970).

[10] Reed, M. and Simon, B., Methods of modern mathematical physics, I, Academic Press, New 
York, London, 1972.

[11] Ruckle, W.H., Sequence spaces, Pitman Publ. Lmt., London, 1981.

[12] Schmüdgen, K., Graded and filtrated topological *-algebras, I. Graded normal topologies, Rep. Math. Phys., 18 (1980), 211-229.

[13] Uhlmann, A., Über die Definition der Quantenfelder nach Wightman und Haag, Wiss. Z. KMU Leipzig, 11 (1962), 213-217.

[14] Wilansky, A., Modern methods in topological vector spaces, McGraw Hill, New York, 1978.

[15] Yngvason, J., On the algebra of test functions for field operators, Comm. Math. Phys., 34 (1973), 315-333. 